\title{
Statistical mechanics of the $N$-point vortex system with random intensities on a bounded domain
}

\section{Mécanique statistique des systèmes de $N$ vortex ponctuels à intensités aléatoires sur un domaine borné}

\author{
Cassio Neri ${ }^{1}$ \\ Instituto de Matemática, Universidade Federal do Rio de Janeiro, Caixa Postal 68530, CEP 21945-970, Rio de Janeiro, Brazil
} Received 5 April 2003; accepted 29 April 2003

Available online 16 December 2003

\begin{abstract}
The system of $N$ point vortices on a bounded domain $\Omega$ is considered under the hypothesis that vortex intensities are independent and identically distributed random variables with respect to a law $P$ supported on a bounded subset of $\mathbb{R}$. It is shown that, in the limit $N \rightarrow+\infty$, the 1 -vortex distribution on $\Omega$ is a minimizer of the free energy functional (a combination of entropy and energy functionals) and is associated to (some) solutions of the following non-linear Poisson Equation (called Mean Field Equation):

$$
\begin{cases}-\Delta u(x)=\left[\iint \mathrm{e}^{-\beta r u(y)} \mathrm{d} y P(\mathrm{~d} r)\right]^{-1} \int r_{1} \mathrm{e}^{-\beta r u(x)} P(\mathrm{~d} r), & \forall x \in \Omega, \\ u(x)=0, & \forall x \in \partial \Omega .\end{cases}
$$

(C) 2004 L'Association Publications de l'Institut Henri Poincaré. Published by Elsevier B.V. All rights reserved

\section{Résumé}

Le systéme de $N$ vortex ponctuels sur un domaine $\Omega$ borné est considéré sous l'hypothèse que les intensités des vortex sont des variables aléatoires indépendentes et identiquement distribuées selon la loi $P$ de support inclus dans un borné de $\mathbb{R}$. On montre que, à la limite quand $N \rightarrow+\infty$, la distribution d'un seul vortex dans $\Omega$ est un minimiseur de la fonctionnelle d'énergie libre (qui est une composition de l'entropie et de l'énergie) et qui est associée à (certaines) solutions de l'équation de Poisson non linéaire (dite de Champ Moyen) (1).

(C) 2004 L'Association Publications de l'Institut Henri Poincaré. Published by Elsevier B.V. All rights reserved
\end{abstract}

Keywords: Statistical mechanics; $N$-point vortex system; Onsager theory; Limits of Gibbs's measures; Entropy; Mean field equation

\footnotetext{
E-mail address: cassio@labma.ufrj.br (C. Neri).

${ }^{1}$ Supported by CNPq grant number 200491/97-0, Brazil.
}

0294-1449/\$ - see front matter (C) 2004 L'Association Publications de l'Institut Henri Poincaré. Published by Elsevier B.V. All rights reserved doi:10.1016/j.anihpc.2003.05.002 


\section{Introduction}

Systems of $N$-point vortices in a smooth, bounded, connected open domain $\Omega \subset \mathbb{R}^{2}$ of Lebesgue measure $|\Omega|$ have been studied with some success since Onsager [15]. This paper studies such systems assuming the vortex intensities to be independent and identically distributed random variables with respect to a law $P$. We assume that $P$ is supported on a bounded subset of $\mathbb{R}$ (for simplicity we shall consider $P$ supported on $[-1,1]$ ).

We follow the approach of Cagliotti et al. [2] who handled the case in which all vortices have intensity equal to 1 . This situation corresponds to the law $P$ being the Dirac measure concentrated at 1 and, hence, our results will generalize some of those in [2].

The first motivation for considering random intensities is to provide a mathematical explanation to certain results which are well known to physicists. An example is the work of Joyce and Montgomery [5] on the statistical mechanics of the so-called neutral systems, which are defined by an equal number of vortices of intensity 1 and -1 . This situation is similar to a particular case in our approach (also called neutral) in which each vortex has intensity either 1 or -1 with probabilities $1 / 2$ in each case.

In Section 2 we introduce the canonical Gibbs measure $\mu^{N}$ associated with the system of $N$ vortices and temperature $N / \beta$. The phase space of the Hamiltonian system is, essentially, $\Omega^{N}$ and thus it has finite measure. In Section 3 we shall show that the problem is well posed for $\beta$ in $(-8 \pi, 8 \pi)$. For $\beta<0$ we mean states of negative temperatures as predicted by Onsager and considered by Joyce and Montgomery [5,6,12]. The length of this interval is connected to the support of $P$.

As it happens in all statistical theories, we expect the results should have more physical meaning when $N$ is large. So we let $N$ go to infinity and look for cluster points of $\left(\mu^{N}\right)_{N>1}$ in the following way: we introduce $\mu_{k}^{N}$ $(k \in \mathbb{N})$, the marginal distribution of $k$ vortices induced by $\mu^{N}$. Then we look for weak cluster points (in $L^{p}$ ) of $\mu_{k}^{N}$ as $N \rightarrow+\infty$ with $k$ fixed. Since for each $k$ we have a cluster point of $\mu_{k}^{N}$, we find sequences $\mu_{*}=\left(\mu_{k}\right)_{k \in \mathbb{N}}$, called weak cluster points of $\left(\mu^{N}\right)_{N>1}$, which we characterize by variational arguments. This is the subject of Section 5 . We remark that $\mu^{N}$ minimizes the free-energy functional $F^{N}$ (which is a composition of two terms: entropy and energy). We define the limit functional $F^{*}$ for which weak cluster points are minima.

As $N$ increases, we expect vortex positions to become independent of one another. This means the following: the field created by vortices converges to a mean field; the $k$-vortex distributions $\mu_{k}^{N}$ "factorize" and, at the limit $N \rightarrow+\infty$, this distribution behaves like a product of $k$ copies of 1 -vortex distributions (this factorization property is called "propagation of chaos".)

Our main tool is the Hewitt-Savage theorem [7]. Using this result, we rewrite the limit problem to show that weak cluster points are averages of product distributions, i.e. $\mu_{k}=\int \rho^{\otimes k} \xi(\mathrm{d} \rho)$, where $\xi$ is a probability measure on the space of 1-vortex distributions. When $\beta>0, \xi$ is a Dirac measure and thus we have the propagation of chaos. On the other hand, for negative temperatures, the propagation depends on several factors $(P, \beta$ and the geometry of the domain). In any case, we can show that in the support of $\xi$ we have only 1 -vortex distributions that are minima of a certain functional $F$.

The mean fields are nothing but the Newton potentials associated with minima of $F$. These potentials are solutions of Eq. (1). This is a semi-linear Poisson equation (with an exponential non-linearity) called Mean Field Equation (MFE for short). In the neutral case, up to a constant, we find the same as that found in [1,4-6,9-13]. This constant is a nonlocal and nonlinear term of MFE (this makes numerical implementation more difficult).

The MFE is studied in Sections 6,8, 9 and 10. We introduce another functional $G$ which acts on the potentials and for which the MFE is the associated Euler-Lagrange equation. We show existence of minimizers for $G$ using the sharp form of Moser-Trundiger inequality [14]. We show that $G$ preserves the minimizers of $F$, i.e. each potential associated with a minimizer of $F$ is a minimizer of $G$ and conversely. We find a relationship between the minimal values of these two functionals.

We are interested in the uniqueness of solutions for the MFE, as this is directly related to the propagation of chaos. If uniqueness holds, then we have a unique minimizer of $F$ and hence $\xi$ is a Dirac measure (concentrated at this point). As we have mentioned before, there is propagation of chaos in a positive temperature state, and this 
is indeed a consequence of the uniqueness of solutions for the MFE, which arises from the strict convexity of the functional $G$.

\section{Notation}

We introduce some notation which will be used in the sequel. Set $\widetilde{\Omega}=\Omega \times[-1,1] . \widetilde{X}=\left(\tilde{x}_{1}, \ldots, \tilde{x}_{N}\right)$ denotes an arbitrary point in $\widetilde{\Omega}^{N}$, where $\tilde{x}_{i}=\left(x_{i}, r_{i}\right)\left(x_{i} \in \Omega\right.$ and $\left.r_{i} \in[-1,1]\right)$. All $r_{i}$ 's are random variables identically distributed with respect to a Borelian probability measure $P$ on $[-1,1]$. On $\widetilde{\Omega}$ we consider the product measure Lebesgue $\times P$. By a.e. we mean almost everywhere with respect to Lebesgue, $P$, or Lebesgue $\times P$ measures without precising which one we are considering.

For all $1 \leqslant k \leqslant N$ and $\widetilde{X} \in \widetilde{\Omega}^{N}$ we set $X=\left(x_{1}, \ldots, x_{N}\right)$ and define $\tilde{X}_{k}=\left(\tilde{x}_{1}, \ldots, \tilde{x}_{k}\right)$ and $\tilde{X}^{N-k}=\left(\tilde{x}_{k+1}\right.$, $\left.\ldots, \tilde{x}_{N}\right)\left(X_{k}\right.$ and $X^{N-k}$ are analogous defined).

For the purpose of integration we set $\mathrm{d} \tilde{x}_{i}=\mathrm{d} x_{i} P\left(\mathrm{~d} r_{i}\right), \mathrm{d} \tilde{X}=\mathrm{d} \tilde{x}_{1} \ldots \mathrm{d} \tilde{x}_{N}$, and $\mathrm{d} X=\mathrm{d} x_{1} \ldots \mathrm{d} x_{N}$. In an obvious way we define $\mathrm{d} \widetilde{X}_{k}, \mathrm{~d} \widetilde{X}^{N-k}, \mathrm{~d} X_{k}$, and $\mathrm{d} X^{N-k}$.

The Hamiltonian of the $N$-point vortex system is given by

$$
H^{N}(\widetilde{X})=\frac{1}{2} \sum_{i \neq j}^{N} r_{i} r_{j} V\left(x_{i}, x_{j}\right),
$$

where $V$ is the Green function of the Poisson equation in $\Omega$ with homogeneous Dirichlet boundary conditions. For simplicity, we set $H=H^{2}$.

As it is classical, $V$ is given by

$$
V\left(x_{1}, x_{2}\right)=-\frac{1}{2 \pi} \log \left|x_{1}-x_{2}\right|+\gamma\left(x_{1}, x_{2}\right),
$$

where $\gamma$ is its regular part, which is bounded from above. We know that $V$ is positive and

$$
\int_{\Omega} V\left(x_{1}, x_{2}\right) \mathrm{d} x_{2} \leqslant C, \quad \forall x_{1} \in \Omega,
$$

for some constant $C$ (depending on $\Omega$ ).

The associated Gibbs measure, with inverse temperature $\beta^{-1}$, is defined by

$$
\mu^{N}(\tilde{X})=\frac{1}{Z(N, \beta)} \mathrm{e}^{-\frac{\beta}{N} H^{N}(\tilde{X})},
$$

where

$$
Z(N, \beta)=\int_{\widetilde{\Omega}^{N}} \mathrm{e}^{-\frac{\beta}{N} H^{N}(\tilde{X})} \mathrm{d} \tilde{X}
$$

is the partition function.

Finally $C$, with or without indices, denotes several positive constants and $1_{A}$ denotes the characteristic function of a set $A$. 


\section{Bounds for the partition function}

First of all, we should find the range of $\beta$ for which $\mu^{N}$ makes sense, i.e. for which the integral in (3) converges. The following proposition yields this result and also provides some bounds for the partition function. These bounds will be useful when we shall let $N$ go to $+\infty$.

Proposition 1. Let $\beta \in(-8 \pi, 8 \pi)$. There exist $C_{1}$ and $C_{2}=C_{2}(\beta)$ such that

$$
C_{1}^{N} \leqslant Z(N, \beta) \leqslant C_{2}^{N}, \quad \forall N \geqslant 2 .
$$

Moreover, $C_{2}$ is uniform on compact subsets of $(-8 \pi, 8 \pi)$.

Proof. Let us fix $\alpha \in(0,8 \pi)$ such that $[-\alpha, \alpha]$ is a compact interval which contains $\beta$. By Hölder's inequality and the boundness of $\gamma$ from above we have

$$
\begin{aligned}
& Z(N, \beta) \leqslant \int_{\Omega^{N}} \prod_{i=1}^{N} \mathrm{e}^{\frac{|\beta|}{2 N}} \sum_{j=1, j \neq i}^{N} V\left(x_{i}, x_{j}\right) \mathrm{d} X \leqslant \prod_{i=1}^{N}\left[\int_{\Omega^{N}} \mathrm{e}^{\frac{\alpha}{2}} \sum_{j=1, j \neq i}^{N} V\left(x_{i}, x_{j}\right) \mathrm{d} X\right]^{\frac{1}{N}} \\
& \leqslant \int_{\Omega} \int_{\Omega^{N-1}} \prod_{j=2}^{N} \frac{\mathrm{e}^{C}}{\left|x_{1}-x_{j}\right|^{\alpha / 4 \pi}} \mathrm{d} X^{N-1} \mathrm{~d} x_{1} \leqslant C^{N-1} \int\left[\int_{\Omega} \frac{1}{\left|x_{1}-x_{2}\right|^{\alpha / 4 \pi}} \mathrm{d} x_{2}\right]^{N-1} \mathrm{~d} x_{1} .
\end{aligned}
$$

The innermost integral is finite since $\alpha / 4 \pi<2$. It follows that $Z(N, \beta) \leqslant C^{N-1}|\Omega| \leqslant C_{2}^{N}$ ( $C_{2}$ depends on $\alpha$ but not on $\beta$ ).

The lower bound is a consequence of Jensen's inequality, the positivity of $V$ and (2). Indeed,

$$
\begin{aligned}
Z(N, \beta) & \geqslant|\Omega|^{N} \exp \left(-\frac{\beta}{2 N|\Omega|^{N}} \sum_{i \neq j}^{N} \int_{\Omega^{N}} r_{i} r_{j} V\left(x_{i}, x_{j}\right) \mathrm{d} X\right) \\
& \geqslant|\Omega|^{N} \exp \left(-\frac{4 \pi(N-1)}{|\Omega|^{2}} \int_{\Omega} \int_{\Omega} V\left(x_{1}, x_{2}\right) \mathrm{d} x_{1} \mathrm{~d} x_{2}\right) \geqslant|\Omega|^{N} \mathrm{e}^{-C(N-1)} \geqslant C_{1}^{N} .
\end{aligned}
$$

This completes the proof.

From now on $\beta$ will be fixed in $(-8 \pi, 8 \pi)$.

\section{Existence of weak cluster points for Gibbs measures}

The elements of the Gibbs sequence $\left(\mu^{N}\right)_{N>1}$ are functions defined on different domains. They are points in different functional spaces. This leads to a problem when looking for limits of this sequence. To overcome this problem we introduce the family of correlation functions $\left(\rho_{k}\right)_{1 \leqslant k \leqslant N}$ of a function $\rho \in L^{1}\left(\widetilde{\Omega}^{N}\right)$, defined by

$$
\rho_{k}\left(\tilde{X}_{k}\right)=\int_{\widetilde{\Omega}^{N-k}} \rho(\tilde{X}) \mathrm{d} \tilde{X}^{N-k}
$$

In the probability jargon, when $\rho$ is a probability density on $\widetilde{\Omega}^{N}, \rho_{k}$ is the marginal density of $\widetilde{X}_{k}$. 
Now, for each $k \in \mathbb{N},\left(\mu_{k}^{N}\right)_{N>k}$ is a sequence on $L^{1}\left(\widetilde{\Omega}^{k}\right)$ and thus we can look for its cluster points. Before finding $L^{p}$ estimates for these sequences we find point-wise ones. First we have the following lemma.

Lemma 2. There exists $C$ (depending only on $\Omega$ but not on $\beta$ ) such that

$$
Z\left(k, \frac{\beta k}{N}\right) \leqslant C^{N-k} Z(N, \beta), \quad \forall k \geqslant 2, \forall N>k .
$$

Proof. It is easy to see that

$$
Z\left(k+1, \frac{\beta(k+1)}{N}\right)=\int_{\widetilde{\Omega}^{k}} \mathrm{e}^{-\frac{\beta}{N} H^{k}\left(\tilde{X}_{k}\right)} f\left(\tilde{X}_{k}\right) \mathrm{d} \widetilde{X}_{k},
$$

where

$$
f\left(\widetilde{X}_{k}\right)=\int_{\widetilde{\Omega}} \mathrm{e}^{-\frac{\beta}{N} \sum_{i=1}^{k} r_{i} r_{k+1} V\left(x_{i}, x_{k+1}\right)} \mathrm{d} \tilde{x}_{k+1} .
$$

By Jensen's inequality, the positivity of $V$ and (2) we have

$$
\begin{aligned}
f\left(\tilde{X}_{k}\right) & \geqslant|\Omega| \exp \left(-\frac{\beta}{|\Omega| N} \sum_{i=1}^{k} \int_{\widetilde{\Omega}} r_{i} r_{k+1} V\left(x_{i}, x_{k+1}\right) \mathrm{d} \tilde{x}_{k+1}\right) \\
& \geqslant|\Omega| \exp \left(-\frac{8 \pi}{|\Omega| N} \sum_{i=1}^{k} \int_{\Omega} V\left(x_{i}, x_{k+1}\right) \mathrm{d} x_{k+1}\right) \geqslant|\Omega| \exp \left(-\frac{8 \pi k C}{N}\right) \geqslant|\Omega| \mathrm{e}^{-8 \pi C}=C,
\end{aligned}
$$

which, with Eq. (4), yields

$$
Z\left(k, \frac{\beta k}{N}\right) \leqslant C Z\left(k+1, \frac{\beta(k+1)}{N}\right) .
$$

By induction we finish the proof.

The next proposition yields point-wise estimates for $\left(\mu_{k}^{N}\right)_{N>k}$.

Proposition 3. There exists $C=C(\beta)$ such that

$$
\mu_{k}^{N}\left(\widetilde{X}_{k}\right) \leqslant C^{k} \mathrm{e}^{-\frac{\beta}{N} H^{k}\left(\widetilde{X}_{k}\right)}, \quad \forall k \geqslant 2, \forall N \text { large enough. }
$$

Proof. Let $N_{0}, N \in \mathbb{N}$ and $r, p, p^{\prime} \in \mathbb{R}$ be such that

- $r>1$ with $\beta r \in(-8 \pi, 8 \pi)$;

- $N_{0}=\min \{N \in \mathbb{N} \mid N>2 k$ and $N /(N-2 k)<r\}$ and $N \geqslant N_{0}$;

- $p=N /(N-2 k)$ and $p^{\prime}=N / 2 k$.

It is easy to see that

$$
\mu_{k}^{N}\left(\widetilde{X}_{k}\right)=\frac{1}{Z(N, \beta)} \mathrm{e}^{-\frac{\beta}{N} H^{k}\left(\widetilde{X}_{k}\right)} \int_{\widetilde{\Omega}^{N-k}} \mathrm{e}^{-\frac{\beta}{N} H^{N-k}\left(\widetilde{X}^{N-k}\right)-\frac{\beta}{N} \sum_{i=1}^{k} \sum_{j=k+1}^{N} H\left(\tilde{x}_{i}, \tilde{x}_{j}\right)} \mathrm{d} \tilde{X}^{N-k} .
$$


By Hölder's inequality the last integral is bounded above by

$$
\left[\int_{\widetilde{\Omega}^{N-k}} \mathrm{e}^{-\frac{\beta p}{N} H^{N-k}\left(\widetilde{X}^{N-k}\right)} \mathrm{d} \widetilde{X}^{N-k}\right]^{1 / p}\left[\int_{\widetilde{\Omega}^{N-k}} \mathrm{e}^{-\frac{\beta p^{\prime}}{N} \sum_{i=1}^{k} \sum_{j=k+1}^{N} H\left(\tilde{x}_{i}, \tilde{x}_{j}\right)} \mathrm{d} \widetilde{X}^{N-k}\right]^{1 / p^{\prime}} .
$$

Since $|\beta| p^{\prime} k / 2 \pi N=|\beta| / 4 \pi<2$, by an analogous argument as in the proof of Proposition 1 , we show that the second term is bounded above by $C^{k}(C=C(\beta))$. We easily see that the first term is equal to $Z(N-k, \beta p(N-$ $k) / N)^{1 / p}$. To finish the proof, we shall show that there exists $C=C(\beta)$ such that

$$
\frac{Z(N-k, \beta p(N-k) / N)^{1 / p}}{Z(N, \beta)} \leqslant C^{k}
$$

By Lemma 2 there exists $C>1$ such that

$$
\frac{Z(N-k, \beta p(N-k) / N)^{1 / p}}{Z(N, \beta)} \leqslant C^{k} \frac{Z(N, \beta p)^{1 / p}}{Z(N, \beta)} .
$$

Again, by Hölder's inequality, we have

$$
Z(N, \beta p)^{1 / p} \leqslant Z(N, \beta r)^{\theta / r} Z(N, \beta)^{1-\theta},
$$

i.e.

$$
\frac{Z(N, \beta p)^{1 / p}}{Z(N, \beta)} \leqslant Z(N, \beta r)^{\theta / r} Z(N, \beta)^{-\theta},
$$

where $\theta \in(0,1)$ is such that $1 / p=\theta / r+(1-\theta)$ which yields $\theta=2 k r /(N(r-1))$.

Since $\beta r \in(-8 \pi, 8 \pi)$, Proposition 1 provides $C_{1}=C_{1}(\beta)$ and $C_{2}=C_{2}(\beta)$ such that $Z(N, \beta r)^{\theta / r} \leqslant C_{2}^{N \theta / r} \leqslant$ $C^{k}$ and $Z(N, \beta)^{-\theta} \leqslant C_{1}^{-N \theta} \leqslant C^{k}$.

Finally, we have the $L^{p}$ estimates:

Corollary 4. Let $p \in[1,+\infty)$. We have $\mu_{k}^{N} \in L^{p}\left(\widetilde{\Omega}^{k}\right)$ for all $k \in \mathbb{N}$ and $N$ large enough. Moreover, there exists $C=C(\beta, p)$ such that

$$
\left\|\mu_{k}^{N}\right\|_{L^{p}} \leqslant C^{k} \quad \forall k \in \mathbb{N}, \forall N \text { large enough. }
$$

Hence, if $p>1$, then there exist $\mu_{k} \in L^{p}\left(\Omega^{k}\right)$ and a subsequence $\left(\mu_{k}^{N_{j}}\right)_{k \in \mathbb{N}}$ such that $\mu_{k}^{N_{j}} \rightarrow \mu_{k}$ weakly in $L^{p}\left(\widetilde{\Omega}^{k}\right)$.

Proof. From Proposition 3, for all $k \geqslant 2$ and $N$ large enough, we have

$$
\int_{\widetilde{\Omega}^{k}}\left|\mu_{k}^{N}\left(\tilde{X}_{k}\right)\right|^{p} \mathrm{~d} \widetilde{X}_{k} \leqslant C^{k p} Z\left(k, \frac{\beta k p}{N}\right) .
$$

But $(\beta k p / N)_{N \geqslant k p}$ is in a compact subset of $(-8 \pi, 8 \pi)$ so, by Proposition 1 , there exists $C$ such that $Z(k, \beta k p / N) \leqslant C^{k}$. It follows that $\left\|\mu_{k}^{N}\right\|_{L^{p}} \leqslant C^{k} C^{k / p} \leqslant C^{k}$ for $N$ large enough.

The set of indices $\left(N_{j}\right)_{j \in \mathbb{N}}$ depends on $k$ and $p$ but, by a diagonal process, we can take the same one for all $k$ and $p$. It holds even for $p=1$ since $\widetilde{\Omega}^{k}$ is of finite measure. In the sequel we shall always note $\mu_{*}=\left(\mu_{k}\right)_{k \in \mathbb{N}}$ as a weak cluster point of $\left(\mu^{N}\right)_{N>1}$, that is,

$$
\mu_{k}^{N_{j}} \rightarrow \mu_{k} \quad \text { weakly in } L^{p}\left(\widetilde{\Omega}^{k}\right), \forall k \in \mathbb{N}, \forall p \in[1,+\infty) .
$$

The sequence of indices will be always noted by $\left(N_{j}\right)_{j \in \mathbb{N}}$. 


\section{Looking for cluster points: a variational way}

We wish to find the weak cluster points of $\left(\mu^{N}\right)_{N>1}$. We shall see that each $\mu^{N}$ is a solution of a variational problem and thus one can ask if the cluster points of $\left(\mu^{N}\right)_{N>1}$ are also solutions of some limit problem (when $N$ goes to $+\infty)$. This is the goal of this section.

We set $D\left(F^{N}\right)=\left\{\rho \in L^{1}\left(\widetilde{\Omega}^{N}\right) \mid \rho \log \rho \in L^{1}\left(\widetilde{\Omega}^{N}\right)\right\}$. For $\rho \in D\left(F^{N}\right)$ we define the following functionals:

$$
\begin{aligned}
& S^{N}(\rho)=\int_{\widetilde{\Omega}^{N}} \rho(\tilde{X}) \log \rho(\tilde{X}) \mathrm{d} \widetilde{X} \quad \text { (entropy), } \\
& E^{N}(\rho)=\frac{1}{N} \int_{\widetilde{\Omega}^{N}} H^{N}(\tilde{X}) \rho(\tilde{X}) \mathrm{d} \widetilde{X} \quad \text { (energy), } \\
& F^{N}(\rho)=S^{N}(\rho)+\beta E^{N}(\rho) \quad \text { (free energy). }
\end{aligned}
$$

Remark that $S^{N}$ is convex and $E^{N}$ is linear thus $F^{N}$ is convex.

By the inequality

$$
s r \leqslant r \log r+\frac{1}{\mathrm{e}} \mathrm{e}^{s}, \quad \forall r \geqslant 0, \forall s \in \mathbb{R},
$$

applied to $r=\rho$ and $s=H^{N} / N$, it follows that $E^{N}(\rho) \in \mathbb{R}$ provided $\rho \in D\left(F^{N}\right)$. Hence $F^{N}$ is well defined from $D\left(F^{N}\right)$ to $\mathbb{R}$.

Remark 5. Whenever $\rho$ is symmetric a simpler expression for $E^{N}$ holds:

$$
E^{N}(\rho)=\frac{N-1}{2} \iint_{\widetilde{\Omega}} H\left(\tilde{x}_{1}, \tilde{x}_{2}\right) \rho_{2}\left(\tilde{x}_{1}, \tilde{x}_{2}\right) \mathrm{d} \tilde{x}_{1} \mathrm{~d} \tilde{x}_{2} .
$$

An important property of entropy is sub-additivity given by the following proposition.

Proposition 6. Let $1 \leqslant k<N$ and $\rho \in D\left(F^{N}\right)$ symmetric such that $\|\rho\|_{L^{1}}=1$. We have

$$
S^{k}\left(\rho_{k}\right)+S^{N-k}\left(\rho_{N-k}\right) \leqslant S^{N}(\rho) .
$$

Proof. It is clear that

$$
S^{k}\left(\rho_{k}\right)+S^{N-k}\left(\rho_{N-k}\right)-S^{N}(\rho)=\int_{\widetilde{\Omega}^{N}} \rho(\tilde{X}) \log \left(\frac{\rho_{k}\left(\tilde{X}_{k}\right) \rho_{N-k}\left(\tilde{X}^{N-k}\right)}{\rho(\widetilde{X})}\right) \mathrm{d} \widetilde{X} .
$$

By Jensen's inequality the last integral is bounded above by

$$
\log \left(\int_{\widetilde{\Omega}^{N}} \rho_{k}\left(\tilde{X}_{k}\right) \rho_{N-k}\left(\tilde{X}^{N-k}\right) \mathrm{d} \tilde{X}\right),
$$

which is null since $\|\rho\|_{L^{1}}=1$. The proposition follows.

Lemma 7. For all $C>0$ the set

$$
M_{C}=\left\{\rho \in D\left(F^{N}\right) \mid \int_{\widetilde{\Omega}^{N}}[\rho(\tilde{X}) \log \rho(\tilde{X})]^{+} \mathrm{d} \tilde{X} \leqslant C\right\}
$$

is weakly compact on $L^{1}\left(\widetilde{\Omega}^{N}\right)$. 
Proof. We remark that $M_{C}$ is convex since $D\left(F^{N}\right)$ and $t \mapsto[t \log t]^{+}$are convex. By Fatou's lemma, $M_{C}$ is strongly closed in $L^{1}\left(\widetilde{\Omega}^{N}\right)$ and thus, by convexity, it is weakly closed.

We have to show that every sequence $\left(\rho_{n}\right)_{n \in \mathbb{N}}$ in $M_{C}$ has a subsequence weakly convergent in $L^{1}\left(\widetilde{\Omega}^{N}\right)$. For $M>1$ and $n \in \mathbb{N}$ we have

$$
\int_{\left\{\rho_{n} \geqslant M\right\}} \rho_{n}(\tilde{X}) \log M \mathrm{~d} \tilde{X} \leqslant \int_{\widetilde{\Omega}^{N}}\left[\rho_{n}(\tilde{X}) \log \rho_{n}(\tilde{X})\right]^{+} \mathrm{d} \tilde{X} \leqslant C .
$$

Hence

$$
\sup _{n \in \mathbb{N}} \int_{\left\{\rho_{n} \geqslant M\right\}} \rho_{n}(\tilde{X}) \mathrm{d} \tilde{X} \leqslant \frac{C}{\log M} \rightarrow 0 \quad \text { as } M \rightarrow+\infty .
$$

The result follows from Dunford-Pettis's theorem.

Here we have our first variational problem:

Theorem 8. We have that $\mu^{N}$ is the unique solution of

$$
\min \left\{F^{N}(\rho) \mid \rho \in D\left(F^{N}\right),\|\rho\|_{L^{1}}=1\right\} .
$$

Proof. We split the proof into two steps: in the first step we shall show that the problem has a solution $\tilde{\mu}$, and in the second step we shall prove that $\tilde{\mu}=\mu^{N}$.

Step 1: Let $\rho \in D\left(F^{N}\right)$ and $t \geqslant 1$ such that $\beta t \in(-8 \pi, 8 \pi)$. From inequality (5), applied to $r=\rho / t$ and $s=-\beta t H^{N} / N$, it follows that

$$
\rho \log \rho+\frac{\beta}{N} H^{N} \rho \geqslant\left(1-\frac{1}{t}\right) \rho \log \rho+\frac{1}{t} \rho \log t-\frac{1}{\mathrm{e}} \mathrm{e}^{-\frac{\beta t}{N} H^{N}} .
$$

In particular, for $t=1$, one has

$$
\rho \log \rho+\frac{\beta}{N} H^{N} \rho+\frac{1}{\mathrm{e}} \mathrm{e}^{-\frac{\beta}{N} H^{N}} \geqslant 0 .
$$

By Fatou's lemma it follows that $F^{N}$ is an l.s.c. functional in the strong topology of $L^{1}\left(\widetilde{\Omega}^{N}\right)$. Hence, by convexity, $F^{N}$ is also l.s.c. in the weak topology of $L^{1}\left(\widetilde{\Omega}^{N}\right)$.

Let $\left(\rho_{n}\right)_{n \in \mathbb{N}} \subset D\left(F^{N}\right)$ be a minimizing sequence of the problem. Taking $t>1$ in (6) and integrating on $\widetilde{\Omega}^{N}$ we obtain

$$
C \geqslant F^{N}\left(\rho_{n}\right) \geqslant\left(1-\frac{1}{t}\right) \int_{\widetilde{\Omega}^{N}} \rho_{n}(\tilde{X}) \log \rho_{n}(\tilde{X}) \mathrm{d} \tilde{X}+\frac{1}{t} \log t-\frac{1}{\mathrm{e}} Z(N, \beta t) .
$$

Hence,

$$
\int_{\widetilde{\Omega}^{N}} \rho_{n}(\tilde{X}) \log \rho_{n}(\tilde{X}) \mathrm{d} \widetilde{X} \leqslant C, \quad \forall n \in \mathbb{N} .
$$

Since the mapping $t \in[0,+\infty) \mapsto t \log t$ is bounded from below, we have shown that there exists $C>0$ such that $\left(\rho_{n}\right)_{n \in \mathbb{N}}$ is in a set $M_{C}$ as in the Lemma 7 and thus it has a subsequence weakly convergent to $\tilde{\mu} \in M_{C}$. Hence, $\tilde{\mu} \in D\left(F^{N}\right)$ and $\|\tilde{\mu}\|_{L^{1}}=1$. By the lower semi-continuity of $F^{N}$ in the weak topology of $L^{1}\left(\widetilde{\Omega}^{N}\right)$, it follows that $\tilde{\mu}$ is a solution of the problem.

Step 2: Formally $\mu^{N}$ is the unique solution of the Euler-Lagrange equation associated to the problem and thus we should have $\tilde{\mu}=\mu^{N}$. But if $\tilde{\mu}$ vanishes on a set of positive measure, then the derivative of $S^{N}$ will have a 
singularity at $\tilde{\mu}$. Therefore, a rigorous proof of this result is not so straightforward. To avoid this problem we set, for $\delta>0$,

$$
\Lambda_{\delta}=\left\{\tilde{X} \in \widetilde{\Omega}^{N} \mid \tilde{\mu}(\tilde{X})>\delta\right\} \quad \text { and } \quad U_{\delta}=\left\{\varphi \in L^{\infty}\left(\widetilde{\Omega}^{N}\right) \mid\|\varphi\|_{L^{\infty}}<\delta / 2\right\} .
$$

We define the following functionals

$$
\begin{aligned}
J_{\delta}: U_{\delta} \rightarrow \mathbb{R} \text { and } G_{\delta}: U_{\delta} \rightarrow \mathbb{R}, \\
\varphi \mapsto F^{N}\left(\tilde{\mu}+1_{\Lambda_{\delta}} \varphi\right), \quad \varphi \mapsto \int_{\widetilde{\Omega}^{N}} 1_{\Lambda_{\delta}}(\tilde{X}) \varphi(\tilde{X}) \mathrm{d} \tilde{X} .
\end{aligned}
$$

We easily see that both functionals are well defined. Clearly $\varphi=0$ is a minimizer of $J_{\delta}$ restricted to the constraint $G_{\delta}(\varphi)=0$. Now, one can easily (and rigorously) derivate the associated Euler-Lagrange equation to show that there exists $C=C(\delta)$ such that $\tilde{\mu}=C \mathrm{e}^{-\frac{\beta}{N} H^{N}}$ almost everywhere in $\Lambda_{\delta}$. But $\Lambda_{\delta} \subset \Lambda_{\delta^{\prime}}$ whenever $\delta^{\prime}<\delta$. Hence the constant $C$ does not depend on $\delta$ and $\tilde{\mu}$ is given by the previous expression on the subset $\Lambda$ of $\Omega^{N}$ where $\tilde{\mu}$ does not vanish. From $\|\tilde{\mu}\|_{L^{1}}=1$ we obtain

$$
C=\left[\int_{\Lambda} \mathrm{e}^{-\frac{\beta}{N} H^{N}(\tilde{X})} \mathrm{d} \widetilde{X}\right]^{-1}
$$

It is easy to show that

$$
\mathrm{e}^{-F^{N}(\tilde{\mu})}=\int_{\Lambda} \mathrm{e}^{-\frac{\beta}{N} H^{N}(\tilde{X})} \mathrm{d} \tilde{X} \quad \text { and } \quad \mathrm{e}^{-F^{N}\left(\mu^{N}\right)}=\int_{\widetilde{\Omega}^{N}} \mathrm{e}^{-\frac{\beta}{N} H^{N}(\tilde{X})} \mathrm{d} \tilde{X} .
$$

Since $F^{N}(\tilde{\mu}) \leqslant F^{N}\left(\mu^{N}\right)$ we should have $\Lambda=\widetilde{\Omega}^{N}$, and thus $\tilde{\mu}=\mu^{N}$.

Remark 9. In the last proof we have shown that $F^{N}$ is l.s.c. in the weak topology of $L^{1}\left(\widetilde{\Omega}^{N}\right)$. Hence, since $\widetilde{\Omega}^{N}$ is of finite measure, $F^{N}$ is l.s.c. in the weak topology of $L^{p}\left(\widetilde{\Omega}^{N}\right)$ for all $p \in[1,+\infty)$. The same result holds for $S^{N}$ (since $S^{N}=F^{N}$ when $\beta=0$ ).

We define the set $D\left(F^{*}\right)$ of all $\rho_{*}=\left(\rho_{k}\right)_{k \in \mathbb{N}} \in \prod_{k=1}^{+\infty} D\left(F^{k}\right)$ which verifies, for all $k \in \mathbb{N}$,

(i) $\left\|\rho_{k}\right\|_{L^{1}}=1$;

(ii) $\rho_{k}$ is symmetric;

(iii) $\rho_{k}\left(\widetilde{X}_{k}\right)=\int_{\widetilde{\Omega}} \rho_{k+1}\left(\widetilde{X}_{k+1}\right) \mathrm{d} \tilde{x}_{k+1}$;

(iv) $\left\|\rho_{k}\right\|_{L^{\infty}\left(\widetilde{\Omega}^{k}\right)} \leqslant C^{k}$ for some constant $C=C\left(\rho_{*}\right)$.

For $\rho_{*} \in D\left(F^{*}\right)$ we define the functionals

$$
\begin{aligned}
& S^{*}\left(\rho_{*}\right)=\lim _{k \rightarrow+\infty} \frac{1}{k} \int_{\widetilde{\Omega}^{k}} \rho_{k}\left(\tilde{X}_{k}\right) \log \rho_{k}\left(\tilde{X}_{k}\right) \mathrm{d} \tilde{X}_{k}=\lim _{k \rightarrow+\infty} \frac{1}{k} S^{k}\left(\rho_{k}\right), \\
& E^{*}\left(\rho_{*}\right)=\frac{1}{2} \int_{\widetilde{\Omega}} \int_{\widetilde{\Omega}} H\left(\tilde{x}_{1}, \tilde{x}_{2}\right) \rho_{2}\left(\tilde{x}_{1}, \tilde{x}_{2}\right) \mathrm{d} \tilde{x}_{1} \mathrm{~d} \tilde{x}_{2}, \\
& F^{*}\left(\rho_{*}\right)=S^{*}\left(\rho_{*}\right)+\beta E^{*}\left(\rho_{*}\right) .
\end{aligned}
$$

For $\rho_{*} \in D\left(F^{*}\right)$ we have, again by (5), that $E^{*}\left(\rho_{*}\right) \in \mathbb{R}$. The property (iii) implies, by induction, that

$$
\rho_{k}\left(\widetilde{X}_{k}\right)=\int_{\widetilde{\Omega}^{N-k}} \rho_{N}(\widetilde{X}) \mathrm{d} \widetilde{X}^{N-k}, \quad \forall k \in \mathbb{N}, \forall N>k .
$$


Hence, by Proposition 6, we have that $\left(S^{k}\left(\rho_{k}\right)\right)_{k \in \mathbb{N}}$ is a sub-additivity sequence and thus the limit defining $S^{*}$ exists but it is possibly infinite. However, by the property (iv), we have the following bounds

$$
\frac{1}{k} \int_{\widetilde{\Omega}^{k}} \rho_{k}\left(\widetilde{X}_{k}\right) \log \rho_{k}\left(\widetilde{X}_{k}\right) \mathrm{d} \widetilde{X}_{k} \leqslant \frac{1}{k} \int_{\widetilde{\Omega}^{k}} \rho_{k}\left(\widetilde{X}_{k}\right) \log C^{k} \mathrm{~d} \widetilde{X}_{k}=\log C .
$$

We conclude that $S^{*}\left(\rho_{*}\right) \in \mathbb{R}$ and thus $F^{*}$ is well defined from $D\left(F^{*}\right)$ to $\mathbb{R}$.

Proposition 10. Let $\rho_{*} \in D\left(F^{*}\right)$ and $\mu_{*}$ be a weak cluster point of $\left(\mu^{N}\right)_{N>1}$. We have the following convergences:

(i) $N^{-1} E^{N}\left(\rho_{N}\right) \rightarrow E^{*}\left(\rho_{*}\right)$ as $N \rightarrow+\infty$;

(ii) $N^{-1} S^{N}\left(\rho_{N}\right) \rightarrow S^{*}\left(\rho_{*}\right)$ as $N \rightarrow+\infty$;

(iii) $N^{-1} F^{N}\left(\rho_{N}\right) \rightarrow F^{*}\left(\rho_{*}\right)$ as $N \rightarrow+\infty$;

(iv) $N_{j}^{-1} E^{N_{j}}\left(\mu^{N_{j}}\right) \rightarrow E^{*}\left(\mu_{*}\right)$ as $j \rightarrow+\infty$;

(v) $N_{j}^{-1} F^{N_{j}}\left(\mu^{N_{j}}\right) \rightarrow F^{*}\left(\mu_{*}\right)$ as $j \rightarrow+\infty$;

(vi) $N_{j}^{-1} S^{N_{j}}\left(\mu^{N_{j}}\right) \rightarrow S^{*}\left(\mu_{*}\right)$ as $j \rightarrow+\infty$;

(vii) $k^{-1} S^{k}\left(\mu_{k}\right) \leqslant \liminf _{j \rightarrow+\infty} k^{-1} S^{k}\left(\mu_{k}^{N_{j}}\right) \leqslant \limsup _{j \rightarrow+\infty} k^{-1} S^{k}\left(\mu_{k}^{N_{j}}\right) \leqslant S^{*}\left(\mu_{*}\right)$.

Proof. (i) follows trivially from symmetry of $\rho_{k}$ and Remark 5 while (ii) is just the definition of $S^{*}$. Clearly, (iii) is consequence of (i) and (ii).

(iv) follows from symmetry of $\mu^{N_{j}}$ and from the weak convergence $\mu_{2}^{N_{j}} \rightarrow \mu_{2}$ in $L^{2}\left(\widetilde{\Omega}^{2}\right)$ (notice that by Proposition 1 we have $\left.H \in L^{2}\left(\widetilde{\Omega}^{2}\right)\right)$.

Let us show (v). Let $k \in \mathbb{N}$ be fixed. For every $j \in \mathbb{N}$, large enough, we can find two integers $m_{j}$ and $n_{j}$ such that $N_{j}=m_{j} k+n_{j}$ and $0<n_{j} \leqslant k$. By sub-additivity of $S^{N}$ we have

$$
\frac{m_{j}}{N_{j}} S^{k}\left(\mu_{k}^{N_{j}}\right)+\frac{1}{N_{j}} S^{n_{j}}\left(\mu_{n_{j}}^{N_{j}}\right) \leqslant \frac{1}{N_{j}} S^{N_{j}}\left(\mu^{N_{j}}\right) .
$$

The function $t \in[0,+\infty) \mapsto t \log t$ is bounded from below and so is the entropy. Hence,

$$
\frac{m_{j}}{N_{j}} S^{k}\left(\mu_{k}^{N_{j}}\right)-\frac{C}{N_{j}} \leqslant \frac{1}{N_{j}} S^{N_{j}}\left(\mu^{N_{j}}\right) .
$$

By adding $\beta N_{j}^{-1} E^{N_{j}}\left(\mu^{N_{j}}\right)$ to both sides of last inequality and by minimality of $F^{N}\left(\mu^{N}\right)$ it follows that

$$
\frac{m_{j}}{N_{j}} S^{k}\left(\mu_{k}^{N_{j}}\right)+\frac{\beta}{N_{j}} E^{N_{j}}\left(\mu^{N_{j}}\right)-\frac{C}{N_{j}} \leqslant \frac{1}{N_{j}} F^{N_{j}}\left(\mu^{N_{j}}\right) \leqslant \frac{1}{N_{j}} F^{N_{j}}\left(\mu_{N_{j}}\right) .
$$

Taking limits as $j \rightarrow+\infty$, from (iii), (iv), weak lower semi-continuity of $S^{k}$ (see Remark 9) and since $m_{j} / N_{j} \rightarrow$ $1 / k$ it follows that

$$
\frac{1}{k} S^{k}\left(\mu_{k}\right)+\beta E^{*}\left(\mu_{*}\right) \leqslant \liminf _{j \rightarrow+\infty} \frac{1}{N_{j}} F^{N_{j}}\left(\mu^{N_{j}}\right) \leqslant \limsup _{j \rightarrow+\infty} \frac{1}{N_{j}} F^{N_{j}}\left(\mu^{N_{j}}\right) \leqslant F^{*}\left(\mu_{*}\right) .
$$

Finally, we let $k \rightarrow+\infty$ and we use (ii) to conclude.

(vi) follows from (iv) and (v).

It remains to show (vii). The first inequality is just the lower semi-continuity of $S^{k}$. The second one is trivial. The last one follows from (vi) and by taking limits, as $j \rightarrow+\infty$, in (7).

Theorem 11. Let $\mu_{*}$ be a weak cluster point of $\left(\mu^{N}\right)_{N>1}$. We have that $\mu_{*}$ is a solution of

$$
\min \left\{F^{*}\left(\rho_{*}\right) \mid \rho_{*} \in D\left(F^{*}\right)\right\} .
$$


Proof. For $\rho_{*} \in D\left(F^{*}\right)$, by minimality of $F^{N_{j}}\left(\mu^{N_{j}}\right)$, we have $F^{N_{j}}\left(\mu^{N_{j}}\right) \leqslant F^{N_{j}}\left(\rho_{N_{j}}\right)$. So the theorem follows from Proposition 10 (iii) and (v).

We recall that the Gibbs measure gives the distribution of vortices on the phase space. So, it is physically reasonable that the more vortices we have the more their positions will be independent from one another. So, at limit $N \rightarrow+\infty$, we can think that the distribution of $k$ vortices $\mu_{k}$ will be induced by a distribution $\mu$ of onevortice, that is, $\mu_{k}\left(\tilde{X}_{k}\right)=\mu\left(\tilde{x}_{1}\right) \ldots \mu\left(\tilde{x}_{k}\right)$. This factorization property is usually called "propagation of chaos". This does not always holds. In fact, $\mu_{k}$ is not a product measure but an average of product measures (in this case we are talking about "partial propagation of chaos"). Partial propagation holds not only for cluster points of $\left(\mu_{k}^{N}\right)_{N>k}$ but for all $\rho_{*} \in D\left(F^{*}\right)$. The property of $\rho_{*}$ which assures this result is the symmetry of all $\rho_{k}$ 's.

By $\mathcal{P}(\widetilde{\Omega})$ we denote the space of all Borelian probabilities on $\widetilde{\Omega}$ endowed with the weak topology. We denote $\mathcal{Q}(\widetilde{\Omega})$ the set of all Borelian probabilities $v$ on $\mathcal{P}(\widetilde{\Omega})$ which are supported on a bounded set of $L^{\infty}(\widetilde{\Omega})$.

Theorem 12. The application which maps each $v \in \mathcal{Q}(\widetilde{\Omega})$ to $\rho_{*} \in D\left(F^{*}\right)$ defined by

$$
\rho_{k}\left(\tilde{X}_{k}\right)=\int_{\mathcal{P}(\widetilde{\Omega})} \rho\left(\tilde{x}_{1}\right) \ldots \rho\left(\tilde{x}_{k}\right) v(\mathrm{~d} \rho), \quad \forall k \in \mathbb{N},
$$

which is equivalent to

$$
\int_{\widetilde{\Omega}^{k}} f\left(\tilde{X}_{k}\right) \rho_{k}\left(\tilde{X}_{k}\right) \mathrm{d} \widetilde{X}_{k}=\int_{\mathcal{P}(\widetilde{\Omega})} \int_{\widetilde{\Omega}^{k}} f\left(\tilde{X}_{k}\right) \rho\left(\tilde{x}_{1}\right) \ldots \rho\left(\tilde{x}_{k}\right) \mathrm{d} \widetilde{X}_{k} v(\mathrm{~d} \rho), \quad \forall f \in L^{1}\left(\widetilde{\Omega}^{k}\right),
$$

is onto.

Proof. Let $\rho_{*} \in D\left(F^{*}\right)$. We can view $\rho_{*}$ as the probability on $\widetilde{\Omega}^{\mathbb{N}}$ for which $\rho_{k}$ is the marginal density of $\widetilde{\Omega}^{k}$ (for all $k \in \mathbb{N}$ ). By the Hewitt-Savage theorem (see Theorem 7.4 of [7]) there exists a unique Borelian probability $v$ on $\mathcal{P}(\widetilde{\Omega})$ such that

$$
\int_{\widetilde{\Omega}^{k}} f\left(\widetilde{X}_{k}\right) \rho_{k}\left(\widetilde{X}_{k}\right) \mathrm{d} \widetilde{X}_{k}=\int_{\mathcal{P}(\widetilde{\Omega})} \int_{\widetilde{\Omega}^{k}} f\left(\widetilde{X}_{k}\right) \rho\left(\mathrm{d} \tilde{x}_{1}\right) \ldots \rho\left(\mathrm{d} \tilde{x}_{k}\right) v(\mathrm{~d} \rho) .
$$

Taking $f\left(\tilde{X}_{k}\right)=g\left(\tilde{x}_{1}\right) \ldots g\left(\tilde{x}_{k}\right)\left(g \in L^{1}(\widetilde{\Omega})\right)$ and recalling that $\left\|\rho_{k}\right\|_{L^{\infty}} \leqslant C^{k}$ we find

$$
\int_{\mathcal{P}(\widetilde{\Omega})}\left[\int_{\widetilde{\Omega}} g\left(\tilde{x}_{1}\right) \rho\left(\mathrm{d} \tilde{x}_{1}\right)\right]^{k} v(\mathrm{~d} \rho) \leqslant C^{k}\|g\|_{L^{1}}^{k}, \quad \forall g \in L^{1}(\widetilde{\Omega}), \forall k \in \mathbb{N} .
$$

Hence,

$$
\left|\int_{\widetilde{\Omega}} g\left(\tilde{x}_{1}\right) \rho\left(\mathrm{d} \tilde{x}_{1}\right)\right| \leqslant C\|g\|_{L^{1}}, \quad v \text {-almost all } \rho \in \mathcal{P}(\widetilde{\Omega}) .
$$

This means that $v$ is supported inside the ball of $L^{\infty}(\widetilde{\Omega})$ of radius $C$ centered at the origin. It follows that $v \in \mathcal{Q}(\widetilde{\Omega})$ and Eq. (9) becomes (8).

Using (8) to $f=H$ we obtain

$$
E^{*}\left(\rho_{*}\right)=\int_{\mathcal{P}(\widetilde{\Omega})} E(\rho) v(\mathrm{~d} \rho), \quad \text { where } E(\rho)=\frac{1}{2} \iint_{\widetilde{\Omega}} H\left(\tilde{x}_{1}, \tilde{x}_{2}\right) \rho\left(\tilde{x}_{1}\right) \rho\left(\tilde{x}_{2}\right) \mathrm{d} \tilde{x}_{1} \mathrm{~d} \tilde{x}_{2} .
$$


In [16] the author shows that

$$
S^{*}\left(\rho_{*}\right)=\int_{\mathcal{P}(\widetilde{\Omega})} S(\rho) v(\mathrm{~d} \rho), \quad \text { where } S(\rho)=\int_{\widetilde{\Omega}} \rho\left(\tilde{x}_{1}\right) \log \rho\left(\tilde{x}_{1}\right) \mathrm{d} \tilde{x}_{1} .
$$

Hence, setting $F=S+\beta E$, we find

$$
F^{*}\left(\rho_{*}\right)=\int_{\mathcal{P}(\widetilde{\Omega})} F(\rho) v(\mathrm{~d} \rho) .
$$

The functionals $E, S$ and $F$ are well defined from $D(F)=D\left(F^{1}\right)$ to $\mathbb{R}$.

Let $\mu_{*} \in D\left(F^{*}\right)$ be a weak cluster point of $\left(\mu^{N}\right)_{N>1}$ and consider the corresponding $\xi \in \mathcal{Q}(\widetilde{\Omega})$ given by Theorem 12 , i.e. $\mu_{*}$ and $\xi$ are related by

$$
\mu_{k}\left(\widetilde{X}_{k}\right)=\int_{\mathcal{P}(\widetilde{\Omega})} \mu\left(\tilde{x}_{1}\right) \ldots \mu\left(\tilde{x}_{k}\right) \xi(\mathrm{d} \mu), \quad \forall k \in \mathbb{N} .
$$

By using Eq. (10) one can rewrite the claim of Theorem 11 to find that $\xi$ is a solution of

$$
\min \left\{\int_{\mathcal{P}(\widetilde{\Omega})} F(\rho) v(\mathrm{~d} \rho) \mid v \in \mathcal{Q}(\widetilde{\Omega})\right\}
$$

Thus we obtain easily the following theorem.

Theorem 13. The functional $F$ is $\xi$-almost all constant on $\operatorname{supp} \xi$ and equal to its minimum value. In other words, $\xi$-almost all $\mu \in \operatorname{supp} \xi$ is a solution of

$$
\min \left\{F(\rho) \mid \rho \in \mathcal{P}(\widetilde{\Omega}) \cap L^{\infty}(\widetilde{\Omega})\right\} .
$$

We finish this section precising what happens if $F$ has a unique minimizer.

Proposition 14. If $\mu$ is the unique minimizer of $F$ on $\mathcal{P}(\widetilde{\Omega}) \cap L^{\infty}(\widetilde{\Omega})$, then $\left(\mu_{k}^{N}\right)_{N>k}$ converges strongly in $L^{p}\left(\widetilde{\Omega}^{k}\right)$ to $\mu^{\otimes k}$, for all $k \in \mathbb{N}$ and for all $p \in[1,+\infty)$.

Proof. Let $k \in \mathbb{N}$ and $p \in[1,+\infty)$. Take a weak cluster point $\mu_{*}$ of $\left(\mu^{N}\right)_{N>1}$ and $\xi$ such that (11) holds. By Theorem 13, $\mu$ is the unique point in the support of $\xi$, i.e. $\xi$ is a Dirac measure supported at $\mu$. Consequently, $\mu_{k}\left(\tilde{X}_{k}\right)=\mu\left(\tilde{x}_{1}\right) \ldots \mu\left(\tilde{x}_{k}\right)$ and

$$
\frac{1}{k} S^{k}\left(\mu_{k}\right)=\frac{1}{k} \int_{\widetilde{\Omega}^{k}} \mu_{k}\left(\widetilde{X}_{k}\right) \log \mu_{k}\left(\tilde{X}_{k}\right) \mathrm{d} \widetilde{X}_{k}=\int_{\widetilde{\Omega}} \mu\left(\tilde{x}_{1}\right) \log \mu\left(\tilde{x}_{1}\right) \mathrm{d} \tilde{x}_{1}=S(\mu) .
$$

We know also that

$$
S^{*}\left(\mu_{*}\right)=\int_{\mathcal{P}(\widetilde{\Omega})} S(\rho) \xi(\mathrm{d} \rho)=S(\mu)
$$

From Proposition 10 (vii) we obtain $S^{k}\left(\mu_{k}^{N_{j}}\right) \rightarrow S^{k}\left(\mu_{k}\right)$. Since $\mu_{k} \in L^{\infty}\left(\widetilde{\Omega}^{k}\right), \mu_{k}^{N_{j}} \rightarrow \mu_{k}$ in $L^{p}\left(\widetilde{\Omega}^{k}\right)$ for all $p \in[1,+\infty)$ and $t \mapsto t \log t$ is a strictly convex function, we conclude that $\mu_{k}^{N_{j}} \rightarrow \mu_{k}$ strongly in $L^{p}\left(\widetilde{\Omega}^{k}\right)$. We have shown that every weak cluster point of $\left(\mu^{N}\right)_{N>1}$ is a strong one and unique. The result follows. 


\section{The mean field equation}

The Theorem 13 can be interpreted in the following way. For each weak cluster point $\mu_{*}$ of $\left(\mu^{N}\right)_{N>1}$ we find a measure $\xi \in \mathcal{Q}(\widetilde{\Omega})$. In this process, the minimality of $F^{*}\left(\mu_{*}\right)$ is carried to $\xi$ in the sense that in the support of $\xi$ we should have only minimizers of $F$. Thus to each weak cluster point of $\left(\mu^{N}\right)_{N>1}$ corresponds an "average" (with respect to $\xi$ ) of minimizers of $F$. This means that it is important to find these minimizers. We shall see in the present section that such minimizers are associated to some solutions of a PDE.

We recall that the potential of $\rho \in L^{\infty}(\widetilde{\Omega})$ is the function $v \in C^{1}(\bar{\Omega}) \cap C_{0}(\Omega)$ given by

$$
v\left(x_{1}\right)=\int_{\widetilde{\Omega}} r_{2} V\left(x_{1}, x_{2}\right) \rho\left(\tilde{x}_{2}\right) \mathrm{d} \tilde{x}_{2}, \quad \forall x_{1} \in \Omega .
$$

Consequently, $v$ is (in the distribution sense) a solution of the problem

$$
\begin{cases}-\Delta v\left(x_{1}\right)=\int_{[-1,1]} r_{1} \rho\left(\tilde{x}_{1}\right) P\left(\mathrm{~d} r_{1}\right), & \forall x_{1} \in \Omega, \\ v\left(x_{1}\right)=0, & \forall x_{1} \in \partial \Omega .\end{cases}
$$

We have another relation between a minimizer of $F$ and its potential which is, formally, the Euler-Lagrange equation for $F$. With this new relation, by a boot-strap argument, we are able to show the regularity of minimizers of $F$ and theirs potentials.

Proposition 15. Let $\mu$ be a minimizer of $F$ on $\mathcal{P}(\widetilde{\Omega}) \cap L^{\infty}(\widetilde{\Omega})$ and $u$ its potential. Then we have

$$
\mu\left(\tilde{x}_{1}\right)=\left[\int_{\widetilde{\Omega}} \mathrm{e}^{-\beta r_{2} u\left(x_{2}\right)} \mathrm{d} \tilde{x}_{2}\right]^{-1} \mathrm{e}^{-\beta r_{1} u\left(x_{1}\right)} .
$$

Proof. We have a technical difficulty similar to the one found in the proof of Theorem 8 since, a priori, $\mu$ can vanish on a set of positive measure. We proceed as in the second step of that proof to obtain

$$
\mu\left(\tilde{x}_{1}\right)=C \mathrm{e}^{-\beta r_{1} u\left(x_{1}\right)} \quad \text { on } \Lambda,
$$

and $\mu=0$ almost everywhere on $\Lambda^{\complement}=\widetilde{\Omega} \backslash \Lambda$, where $\Lambda=\left\{\tilde{x}_{1} \in \widetilde{\Omega} \mid \mu\left(\tilde{x}_{1}\right)>0\right\}$ and $C=\left[\int_{\Lambda} \mathrm{e}^{-\beta r_{2} u\left(x_{2}\right)} \mathrm{d} \tilde{x}_{2}\right]^{-1}$.

To finish the proof, we shall show, by contradiction, that $a=\left|\Lambda^{\complement}\right|=0$. If not, for $\delta>0$ small and $\tilde{x}_{1} \in \widetilde{\Omega}$, we set

$$
\rho=\frac{\mu+\delta 1_{\Lambda^{\complement}}}{1+\delta a}
$$

We easily see that that $\rho \in \mathcal{P}(\widetilde{\Omega}) \cap L^{\infty}(\widetilde{\Omega})$. By simple (but tedious) computations we find a constant $C>0$, independent of $\delta$, such that

$$
F(\rho) \leqslant F(\mu)+C \delta(1+\log \delta) .
$$

Thus, choosing $\delta$ small enough, we have $F(\rho)<F(\mu)$. Which contradicts the minimality of $F(\mu)$. 
As we have announced before, by a boot-strap argument, we find one of our main results:

Theorem 16. For $\xi$-almost all $\mu \in \operatorname{supp} \xi$ its potential $u$ is in $C^{\infty}(\bar{\Omega})$ and satisfies the following equation (called Mean Field Equation, or MFE for short):

$$
\begin{cases}-\Delta u\left(x_{1}\right)=\left[\int_{\widetilde{\Omega}} \mathrm{e}^{-\beta r_{2} u\left(x_{2}\right)} \mathrm{d} \tilde{x}_{2}\right]^{-1} \int_{[-1,1]} r_{1} \mathrm{e}^{-\beta r_{1} u\left(x_{1}\right)} P\left(\mathrm{~d} r_{1}\right), & \forall x_{1} \in \Omega, \\ u\left(x_{1}\right)=0, & \forall x_{1} \in \partial \Omega .\end{cases}
$$

\section{Two examples}

Before studying solutions of MFE, let us give two examples to see how the equation looks like.

First we consider the case in which $P$ is the Dirac measure supported at 1 , which means that all vortices have certainly intensity 1 . Let $u$ be as in Theorem 16. We calculate both integrals in (13) to conclude that $u$ satisfies

$$
\begin{cases}-\Delta u\left(x_{1}\right)=\left[\int_{\Omega} \mathrm{e}^{-\beta u\left(x_{2}\right)} \mathrm{d} x_{2}\right]^{-1} \mathrm{e}^{-\beta u\left(x_{1}\right)}, & \forall x_{1} \in \Omega, \\ u\left(x_{1}\right)=0, & \forall x_{1} \in \partial \Omega .\end{cases}
$$

This case was studied in $[2,3,8]$.

Now we take $P$ as the half sum of the Dirac measures supported at -1 and 1 , that is, each vortex has intensity -1 or 1 , with probability $1 / 2$ in each case. As before, we show that if $u$ is as in Theorem 16 , then it verifies

$$
\begin{cases}-\Delta u\left(x_{1}\right)=-\left[\int_{\Omega} \operatorname{ch}\left(\beta u\left(x_{2}\right)\right) \mathrm{d} x_{2}\right]^{-1} \operatorname{sh}\left(\beta u\left(x_{1}\right)\right), & \forall x_{1} \in \Omega, \\ u\left(x_{1}\right)=0, & \forall x_{1} \in \partial \Omega .\end{cases}
$$

This case is called neutral and it was treated in [1,4-6,9-13].

\section{The mean field equation: preliminary results}

The Theorem 16 show us that it is important to study the mean field equation in details. We shall follow the standard technique of introducing a functional $G$ for which the Euler-Lagrange equation is MFE, and look for its minimizers. Two questions come up:

(i) MFE can have non-physical solutions, i.e. solutions who are not potentials of minimizers of $F$.

(ii) MFE can have (physical) solutions who are not minimizers (but only critical points) of $G$. Since we are interested only in minimizers we can loose some solutions. Similarly, $G$ may have non-physical minimizers.

We shall see that the second problem never arises since each potential of a minimizer of $F$ is a minimizer of $G$ and conversely. Similarly, for positive temperatures, the first problem does not arise. However, we shall see an example in which MFE has non-physical solutions.

When $\beta=0$ the problem is trivial, since it is linear (moreover it has no physical interest). The problem changes with respect to the sign of $\beta$. In this section we make some considerations for both cases which will be studied separately in the next two sections. 
The functional $G$ is defined by

$$
G(v)=\frac{1}{2}\|\nabla v\|_{L^{2}}^{2}+\frac{1}{\beta} \log \left(\int_{\widetilde{\Omega}} \mathrm{e}^{-\beta r_{1} v\left(x_{1}\right)} \mathrm{d} \tilde{x}_{1}\right) .
$$

This functional is well defined from $H_{0}^{1}(\Omega)$ to $\mathbb{R}$, by next lemma which, is a consequence of Trundiger-Moser's inequality in its sharp form [14]. We easily see that critical points of $G$ are solutions of MFE.

Lemma 17. There exists a constant $C$ such that

$$
\int_{\widetilde{\Omega}} \mathrm{e}^{-\beta r_{1} v\left(x_{1}\right)} \mathrm{d} \tilde{x}_{1} \leqslant C \exp \left(\frac{\beta^{2}}{16 \pi}\|\nabla v\|_{L^{2}}^{2}\right), \quad \forall \beta \in \mathbb{R}, \forall v \in H_{0}^{1}(\Omega) .
$$

Proof. Let $\beta \in \mathbb{R}$ and $v \in H_{0}^{1}(\Omega)$ be nonzero. By Young's inequality we have

$$
-\beta r_{1} v\left(x_{1}\right) \leqslant \frac{|\beta|\|\nabla v\|_{L^{2}}}{(8 \pi)^{1 / 2}} \cdot \frac{(8 \pi)^{1 / 2} v\left(x_{1}\right)}{\|\nabla v\|_{L^{2}}} \leqslant \frac{\beta^{2}\|\nabla v\|_{L^{2}}^{2}}{16 \pi}+4 \pi\left|\frac{v\left(x_{1}\right)}{\|\nabla v\|_{L^{2}}}\right|^{2} .
$$

Hence,

$$
\mathrm{e}^{-\beta r_{1} v\left(x_{1}\right)} \leqslant \exp \left(\frac{\beta^{2}}{16 \pi}\|\nabla v\|_{L^{2}}^{2}\right) \exp \left(4 \pi\left|\frac{v\left(x_{1}\right)}{\|\nabla v\|_{L^{2}}}\right|^{2}\right) .
$$

By integrating on $\widetilde{\Omega}$ and applying Trundiger-Moser's inequality yields the result.

As a general result on minimizers of $G$ we have the following.

Theorem 18. Let $\beta \neq 0$. If there exists a bounded minimizing sequence for $G$, then the problem

$$
\min \left\{G(v) \mid v \in H_{0}^{1}(\Omega)\right\}
$$

has a solution.

Proof. Let $\left(u_{n}\right)_{n \in \mathbb{N}} \subset H^{1}(\Omega)$ be a bounded minimizing sequence for $G$. Up to a subsequence, we can suppose

$$
\begin{array}{ll}
u_{n} \rightarrow u & \text { weakly in } H_{0}^{1}(\Omega), \\
u_{n} \rightarrow u & \text { a.e. on } \Omega .
\end{array}
$$

Since $\left(\left\|\nabla u_{n}\right\|_{L^{2}}\right)_{n \in \mathbb{N}}$ is bounded, by Trudinger-Moser inequality there exist $\eta>0$ such that $\left(\mathrm{e}^{\eta u_{n}^{2}}\right)_{n \in \mathbb{N}}$ is bounded in $L^{1}(\Omega)$.

Let us show that $\mathrm{e}^{-\beta r_{1} u_{n}\left(x_{1}\right)} \rightarrow \mathrm{e}^{-\beta r_{1} u\left(x_{1}\right)}$ in $L^{1}(\widetilde{\Omega})$. Notice that we have already point-wise convergence. By Egoroff's theorem, for all $\varepsilon>0$, there exists a set $A \subset \widetilde{\Omega}$ of measure at most $\varepsilon$ such that $u_{n}$ converges uniformly to $u$ on $\widetilde{\Omega} \backslash A$. Thus, for $n \in \mathbb{N}$ large enough, we have

$$
\int_{\widetilde{\Omega}}\left|\mathrm{e}^{-\beta r_{1} u_{n}\left(x_{1}\right)}-\mathrm{e}^{-\beta r_{1} u\left(x_{1}\right)}\right| \mathrm{d} \tilde{x}_{1} \leqslant|\Omega| \varepsilon+\int_{A} \mathrm{e}^{-\beta r_{1} u_{n}\left(x_{1}\right)} \mathrm{d} \tilde{x}_{1}+\int_{A} \mathrm{e}^{-\beta r_{1} u\left(x_{1}\right)} \mathrm{d} \tilde{x}_{1} .
$$

It remains to show that the last two terms go to 0 as $\varepsilon \rightarrow 0$. But this is a simple consequence of the bounds of $\left(\mathrm{e}^{\eta u_{n}^{2}}\right)_{n \in \mathbb{N}}$ in $L^{1}(\Omega)$. Hence

$$
\frac{1}{\beta} \log \left(\int_{\widetilde{\Omega}} \mathrm{e}^{-\beta r_{1} u_{n}\left(x_{1}\right)} \mathrm{d} \tilde{x}_{1}\right) \rightarrow \frac{1}{\beta} \log \left(\int_{\widetilde{\Omega}} \mathrm{e}^{-\beta r_{1} u\left(x_{1}\right)} \mathrm{d} \tilde{x}_{1}\right) .
$$


From which, together with the weak lower semi-continuity of the norm, we obtain

$$
G(u) \leqslant \liminf _{n \rightarrow+\infty} G\left(u_{n}\right)=\inf \left\{G(v) \mid v \in H_{0}^{1}(\Omega)\right\} .
$$

Consequently, $u$ is a minimizer of $G$.

\section{Mean field equation: positive temperatures states}

In this section we suppose $\beta>0$. It is easy to show that the functional $G$ is strictly convex, so it has at most one minimizer. To obtain the existence of a minimizer, by Theorem 18 it is enough to show that there exists a bounded minimizing sequence.

Proposition 19. Every minimizing sequence for $G$ is bounded.

Proof. Let $\left(u_{n}\right)_{n \in \mathbb{N}} \in H_{0}^{1}(\Omega)$ be a minimizing sequence for $G$. By Jensen's, Hölder's and Poincaré's inequalities we obtain

$$
\begin{aligned}
\int_{\widetilde{\Omega}} \mathrm{e}^{-\beta r_{1} u_{n}\left(x_{1}\right)} \mathrm{d} \tilde{x}_{1} & \geqslant|\Omega| \exp \left(-\frac{\beta}{|\Omega|} \int_{\Omega}\left|u_{n}\left(x_{1}\right)\right| \mathrm{d} x_{1}\right) \\
& \geqslant|\Omega| \exp \left[-\frac{\beta}{|\Omega|^{1 / 2}}\left\|u_{n}\right\|_{L^{2}}\right] \geqslant|\Omega| \exp \left(-\frac{\beta C_{1}}{|\Omega|^{1 / 2}}\left\|\nabla u_{n}\right\|_{L^{2}}\right),
\end{aligned}
$$

where $C_{1}$ is the constant given by the Poincaré inequality. Therefore

$$
G\left(u_{n}\right)=\frac{1}{2}\left\|\nabla u_{n}\right\|_{L^{2}}^{2}+\frac{1}{\beta} \log \left(\int_{\widetilde{\Omega}} \mathrm{e}^{-\beta r_{1} u_{n}\left(x_{1}\right)} \mathrm{d} \tilde{x}_{1}\right) \geqslant \frac{1}{2}\left\|\nabla u_{n}\right\|_{L^{2}}^{2}-\frac{C_{1}}{|\Omega|^{1 / 2}}\left\|\nabla u_{n}\right\|_{L^{2}}+\frac{1}{\beta} \log |\Omega| .
$$

The result follows.

By stricty convexity, the minimizer of $G$ is unique and it is also the unique solution of MFE. Hence, it is the potential of the unique minimizer of $F$. By uniqueness, none of the problems reported on Section 8 arises. We remark that in positive temperature states we have always chaos propagation.

\section{Mean field equation: negative temperatures states}

In this section we assume $\beta<0$.

Proposition 20. There exists $C$ such that

$$
G(v) \geqslant \frac{1}{\beta} \log C \quad \forall \beta \in[-8 \pi, 0), \forall v \in H_{0}^{1}(\Omega) .
$$

Moreover, if $\beta=-8 \pi$ and $P(\{-1,1\})=0$ or if $\beta>-8 \pi$, then every minimizing sequence is bounded.

Proof. Let $\beta \in[-8 \pi, 0)$ and $v \in H_{0}^{1}(\Omega)$. Lemma 17 yields a constant $C$ such that

$$
\log \left(\int_{\widetilde{\Omega}} \mathrm{e}^{-\beta r_{1} v\left(x_{1}\right)} \mathrm{d} \tilde{x}_{1}\right) \leqslant \log C+\frac{\beta^{2}\|\nabla v\|_{L^{2}}^{2}}{16 \pi} .
$$


Therefore

$$
G(v) \geqslant \frac{1}{2}\left(1+\frac{\beta}{8 \pi}\right)\|\nabla v\|_{L^{2}}^{2}+\frac{1}{\beta} \log C \geqslant \frac{1}{\beta} \log C .
$$

This proves the first claim and also that every minimizing sequence is bounded provided $\beta>-8 \pi$. There only remains to prove that, if $\beta=-8 \pi, P(\{-1,1\})=0$ and $\left(u_{n}\right)_{n \in \mathbb{N}} \subset H_{0}^{1}(\Omega)$ is a minimizing sequence of $G$, then $\left(u_{n}\right)_{n \in \mathbb{N}}$ is bounded in $H_{0}^{1}(\Omega)$.

We remark that

$$
G\left(u_{n}\right)+\frac{1}{8 \pi} \log \left(\int_{\widetilde{\Omega}} \mathrm{e}^{8 \pi r_{1} u_{n}\left(x_{1}\right)} \mathrm{d} \tilde{x}_{1}\right)=\frac{1}{2}\left\|\nabla u_{n}\right\|_{L^{2}}^{2} .
$$

So we only have to show that there exists $C$ such that

$$
\int_{\widetilde{\Omega}} \mathrm{e}^{8 \pi r_{1} u_{n}\left(x_{1}\right)} \mathrm{d} \tilde{x}_{1} \leqslant C, \quad \forall n \in \mathbb{N} .
$$

We proceed by contradiction supposing that the above sequence goes to $+\infty$ with $n$. Hence

$$
\int_{\Omega} \mathrm{e}^{8 \pi\left|u_{n}\left(x_{1}\right)\right|} \mathrm{d} x_{1} \rightarrow+\infty \quad \text { as } n \rightarrow+\infty .
$$

Setting $f(t)=\int_{[-1,1]} \mathrm{e}^{8 \pi r_{1} t} P\left(\mathrm{~d} r_{1}\right)$ we easily show that

$$
f(t) \leqslant \mathrm{e}^{8 \pi r|t|}+P([-1,-r] \cup[r, 1]) \mathrm{e}^{8 \pi|t|}, \quad \forall r \in(0,1) .
$$

But $\mathrm{e}^{8 \pi|t|}$ dominates $\mathrm{e}^{8 \pi r|t|}$, when $r<1$ and $|t| \rightarrow+\infty$. Thus, there exists a constant $C=C(r)$ such that

$$
f(t) \leqslant C+2 P([-1,-r] \cup[r, 1]) \mathrm{e}^{8 \pi|t|}, \quad \forall t \in \mathbb{R} .
$$

Consequently,

$$
\varepsilon_{n}=\frac{\int_{\widetilde{\Omega}} \mathrm{e}^{8 \pi r_{1} u_{n}\left(x_{1}\right)} \mathrm{d} \tilde{x}_{1}}{\int_{\Omega} \mathrm{e}^{8 \pi\left|u_{n}\left(x_{1}\right)\right|} \mathrm{d} x_{1}} \leqslant \frac{C}{\int_{\Omega} \mathrm{e}^{8 \pi\left|u_{n}\left(x_{1}\right)\right|} \mathrm{d} x_{1}}+2 P([-1,-r] \cup[r, 1]) .
$$

Taking the limits $n \rightarrow+\infty$ and $r \rightarrow 1$, by (16) and the hypothesis on $P$ we conclude that $\varepsilon_{n} \rightarrow 0$ as $n \rightarrow+\infty$. Without any loose, we suppose $\varepsilon_{n} \leqslant 1$ for all $n \in \mathbb{N}$. Finally we have

$$
\begin{aligned}
G\left(u_{n}\right) & =\frac{1}{2}\left\|\nabla u_{n}\right\|_{L^{2}}^{2}-\frac{1}{8 \pi} \log \left(\int_{\widetilde{\Omega}} \mathrm{e}^{8 \pi r_{1} u_{n}\left(x_{1}\right)} \mathrm{d} \tilde{x}_{1}\right) \geqslant \frac{1}{2}\left\|\nabla\left|u_{n}\right|\right\|_{L^{2}}^{2}-\frac{1}{8 \pi} \log \left(\varepsilon_{n} \int_{\Omega} \mathrm{e}^{8 \pi\left|u_{n}\left(x_{1}\right)\right|} \mathrm{d} x_{1}\right) \\
& \geqslant-\frac{1}{8 \pi} \log C-\frac{1}{8 \pi} \log \varepsilon_{n} \rightarrow+\infty \quad \text { as } n \rightarrow+\infty,
\end{aligned}
$$

which is a contradiction since $\left(u_{n}\right)_{n \in \mathbb{N}}$ is a minimizing sequence.

As a consequence of last proposition and Theorem 18 we have the existence of minimizers of $G$.

Before stating the main result of this section, which says that the second problem stated in the begining of Section 8 does not arise, we prove the following lemma.

Lemma 21. Let $\mu \in L^{\infty}(\widetilde{\Omega})$ and $u$ be its potential. We have

$$
E(\mu)=\frac{1}{2}\|\nabla u\|_{L^{2}}^{2} .
$$


Moreover, if $\mu$ and $u$ verify (12) then

$$
S(\mu)=-\log \left(\int_{\widetilde{\Omega}} \mathrm{e}^{-\beta r_{1} u\left(x_{1}\right)} \mathrm{d} \tilde{x}_{1}\right)-\beta\|\nabla u\|_{L^{2}}^{2} \quad \text { and } \quad F(\mu)=-\beta G(u) .
$$

Proof. Since $u$ is the potential of $\mu$ we have

$$
\int_{\Omega}\left|\nabla u\left(x_{1}\right)\right|^{2} \mathrm{~d} x_{1}=\int_{\widetilde{\Omega}} r_{1} \mu\left(\tilde{x}_{1}\right) u\left(x_{1}\right) \mathrm{d} \tilde{x}_{1},
$$

and thus, by definition of potential,

$$
\frac{1}{2}\|\nabla u\|_{L^{2}}^{2}=\frac{1}{2} \iint_{\widetilde{\Omega}} r_{\widetilde{\Omega}} r_{2} V\left(x_{1}, x_{2}\right) \mu\left(x_{1}\right) \mu\left(x_{2}\right) \mathrm{d} \tilde{x}_{1} \mathrm{~d} \tilde{x}_{2}=E(\mu) .
$$

Using (12) we obtain

$$
\begin{aligned}
S(\mu) & =\int_{\widetilde{\Omega}} \mu\left(\tilde{x}_{1}\right) \log \left(\left[\int_{\widetilde{\Omega}} \mathrm{e}^{-\beta r_{2} u\left(x_{2}\right)} \mathrm{d} \tilde{x}_{2}\right]^{-1} \mathrm{e}^{-\beta r_{1} u\left(x_{1}\right)}\right) \mathrm{d} \tilde{x}_{1} \\
& =-\log \left(\int_{\widetilde{\Omega}} \mathrm{e}^{-\beta r_{1} u\left(x_{1}\right)} \mathrm{d} \tilde{x}_{1}\right)-\beta \int_{\Omega} r_{1} \mu\left(\tilde{x}_{1}\right) u\left(x_{1}\right) \mathrm{d} \tilde{x}_{1} .
\end{aligned}
$$

Again, since $u$ is the potential of $\mu$, we have that the last term above is equal to $-\beta\|\nabla u\|_{L^{2}}^{2}$. The last equality, namely $F(\mu)=-\beta G(u)$, follows from the definition of $G$ and the equations for $E$ and $S$ just proved.

Theorem 22. Let $\mu$ be a minimizer of $F$ and $u$ its potential. Then $u$ is a minimizer of $G$. On the other hand, if $v$ is a minimizer of $G$, then $v \in C^{\infty}(\bar{\Omega}) \cap C_{0}(\Omega)$ and $\rho$ defined by

$$
\rho\left(\tilde{x}_{1}\right)=\left[\int_{\widetilde{\Omega}} \mathrm{e}^{-\beta r_{2} v\left(x_{2}\right)} \mathrm{d} \tilde{x}_{2}\right]^{-1} \mathrm{e}^{-\beta r_{1} v\left(x_{1}\right)}
$$

is a minimizer of $F$.

Proof. By Proposition 15 and Lemma 21 we have $F(\mu)=-\beta G(u)$.

Since $v$ is a critical point of $G$, by elliptic regularity it is a smooth solution of MFE, i.e.

$$
-\Delta v\left(x_{1}\right)=\left[\int_{\widetilde{\Omega}} \mathrm{e}^{-\beta r_{2} v\left(x_{2}\right)} \mathrm{d} \tilde{x}_{2}\right]^{-1} \int_{[-1,1]} r_{1} \mathrm{e}^{-\beta r_{1} v\left(x_{1}\right)} P\left(\mathrm{~d} r_{1}\right) .
$$

Therefore, by (17), $v$ is the potential of $\rho$. Again by Lemma 21 and (17) we have $F(\rho)=-\beta G(v)$.

But $F(\mu) \leqslant F(\rho)$ and $G(v) \leqslant G(u)$, hence, since $\beta<0$, we have $F(\rho)=F(\mu)$ and $G(u)=G(v)$. The conclusion follows.

Now we give an example of a "non-physical" solution. We start by an easy remark: MFE has the trivial null solution if, and only if, $P$ has null average, i.e.

$$
\int_{[-1,1]} r_{1} P\left(\mathrm{~d} r_{1}\right)=0
$$


It represents a uniform distribution of vortices on $\Omega$.

Now consider the neutral case on the square $\Omega=[0,1] \times[0,1]$. It is straightforward that in this case MFE has the trivial null solution. Let us shall show that it is not a physical one. Take $v \in H_{0}^{1}(\Omega)$ an eigenvector of $-\Delta$ associated to its first eigenvalue $\lambda_{1}$ and such that $\|v\|_{L^{2}}=1$. It is a classical fact that $\|\nabla v\|_{L^{2}}^{2}=\lambda_{1}=2 \pi^{2}$. We take a second order Taylor expansion of $G(t v)$ in a neighborhood of $t=0$ and, by straightforward computations, we obtain

$$
G(t v)=G(0)+t G^{\prime}(0) v+\frac{t^{2}}{2}\left\langle G^{\prime \prime}(0) v, v\right\rangle+\mathrm{o}\left(t^{2}\right)=G(0)+\frac{t^{2}\left(2 \pi^{2}+\beta\right)}{2}+\mathrm{o}\left(t^{2}\right) .
$$

Hence, choosing $\beta \in\left(-8 \pi,-2 \pi^{2}\right)$ and $t$ small enough, we have $G(t v)<G(0)$ and thus 0 is not a minimizer of $G$.

\section{Acknowledgements}

I would like to thank P.-L. Lions who introduced me this subject during my doctorate studies at Université Paris IX-Dauphine.

I am gratefull to H. Bursztyn and R. Rosa for the revision of the manuscript and usefull suggestions.

\section{References}

[1] D.L. Book, S. Fisher, B.E. McDonald, Steady-state distributions of interacting discrete vortices, Phys. Rev. Lett. 34 (1) (1975) 4-8.

[2] E. Caglioti, P.-L. Lions, C. Marchioro, M. Pulvirenti, A special class of stationary flows for two-dimensional Euler equations: a statistical mechanics description, Comm. Math. Phys. 143 (3) (1992) 501-525.

[3] E. Caglioti, P.-L. Lions, C. Marchioro, M. Pulvirenti, A special class of stationary flows for two-dimensional Euler equations: a statistical mechanics description II, Comm. Math. Phys. 174 (2) (1995) 229-260.

[4] H.H. Chen, Y.C. Lee, A.C. Ting, Exact solutions of a nonlinear boundary value problem: the vortices of the two-dimensional sinh-Poisson equation, Phys. D 26 (1987) 37-66.

[5] G. Joyce, D. Montgomery, Negative temperature states for the two-dimensional guiding-center plasma, J. Plasma Phys. 10 (1) (1973) $107-121$.

[6] G. Joyce, D. Montgomery, Statistical mechanics of "negative temperature" states, Phys. Fluids 17 (6) (1974) $1139-1145$.

[7] E. Hewitt, L.J. Savage, Symmetric measures on Cartesian products, Trans. Amer. Math. Soc. 80 (1955) $470-501$.

[8] M.K.-H. Kiessling, Statistical mechanics of classical particles with logarithmic interactions, Comm. Pure Appl. Math. 46 (1) (1993) $27-56$.

[9] T.S. Lundgren, Y.B. Pointin, Statistical mechanics of two-dimensional vortices in a bounded container, Phys. Fluids 19 (10) (1973) 14591470.

[10] T.S. Lundgren, Y.B. Pointin, Statistical mechanics of two-dimensional vortices, J. Stat. Phys. 17 (5) (1977) $323-355$.

[11] B.E. McDonald, Numerical calculation of non unique solutions of a two-dimensional sinh-Poisson equation, J. Comp. Phys. 16 (1974) 360-370.

[12] D. Montgomery, Two-dimensional vortex motion and "negative temperatures", Phys. Lett. A 39 (1972) 7-8.

[13] D. Montgomery, D. Tappert, Conductivity of a two-dimensional guiding center plasma, Phys. Fluids 15 (1972) $683-687$.

[14] J. Moser, A sharp form of an inequality by N. Trudinger, Indiana Univ. Math. J. 20 (1970/71) 1077-1092.

[15] L. Onsager, Statistical hydrodynamics, Nuovo Cimento (9) 6 (2) (1949) 279-287, Supplemento (Convegno Internazionale di Meccanica Statistica);

The collected works of Lars Onsager, in: P.C. Hemmer, H. Holden, S. Kjelstrup Ratkje (Eds.), World Scientific Series in 20th Century Physics, vol. 17, World Scientific, River Edge, 1996.

[16] D. Ruelle, Statistical Mechanics: Rigorous Results, W. A. Benjamin, New York, 1969. 